\title{
Performance analyzing on aviation Air-ground Data Link
}

\author{
Xu Jianyuan \\ shanghai aircraft design research institute, comac, \\ shanghai, china
}

\author{
Deng Xueyun \\ Shanghai Aircraft Design Research Institute, COMAC, \\ Shanghai, China
}

\author{
Cao Li \\ Nanjing University of Aeronautics and Astronautics, \\ Nanjing, China
}

\begin{abstract}
As the standard equipment of the commercial aircrafts, air-ground data link system is widely used in modern aviation transport. However, its validity was unsatisfactory in our testing, especially in error of transmission. In this paper, some influencing factors during air-ground data transmitting were analyzed from the signal transmission. Considering ACARS was based on ARINC-618 protocol, a fundamental study in this article on transmission signal and demodulating signal of the ground air data link system was done on the basis of ACARS signal generated by the transient aircrafts.
\end{abstract}

Keywords-ACARS, Very High Frequency (VHF), Signal Analysis

\section{INTRODUCTION}

As the rapid development of the aviation industry, the modern air transport aircraft is becoming more and more digital. A large number of flight data and information can be collected through various methods and recorded. Through the air-ground data link, real-time data communication can be enabled while airplane is in flight. As a complement and extension of Flight Data Recorder (FDR), the air-ground data link can continuously transmit aircraft performance and navigation data in real time to support the remote monitoring of aircraft performance. Also, it can provide the efficient means for the second dispatch and control of taking off and landing for airlines. Currently, as the standard configuration of large civil aircraft, air-ground data link system is used more and more common in flight. Since 2005 , CAAC has forced the aircraft with more than 100 seats installed to use ACARS system [1].

ACARS system changes the air-ground communications methods from voice mode used in the past to both voice and data modes used currently. This led to great revolution in air-ground communication system, whose value has been accepted by international civil aviation organization [2]. The availability of $99.999 \%$ and convenient transimission mode make the ACARS become the most trusted air-ground communication system in civil aviation field [3]. This kind of air-ground data link network has been constructed and used in the main countries and regions of the world, including China.
At the same time, air-ground data link can provide the real-time communication means which can be used to data acquisition, monitoring and flight management in the aircraft life cycle. The data can be used to onboard function test, flight test and maintenance, etc. Therefore, ACARS technology research can be directly applied to the onboard function test and the flight test data analysis. The related technologies also can be used to support the real-time remote monitoring of aircraft condition, maintenance activities and flight quality monitoring.

It can be achieved optimal function according to different application requirement from airlines, such as identifying the unusual flight status, providing detailed reports of engine parameters, providing comprehensive maintenance and repair program, providing accurate weather reports [4]. In addition, remote monitoring in real time is the development direction of modern civil aviation. It can be used to improve the efficiency of maintenance crew, save cost, as well as provide an important means to ensure the safety of the aircraft. It is not only the mark of the development of status monitoring technology, but also enhance the security of airlines and bring them enormous benefits [5]. The ACARS is an important foundation for realizing remote real-time monitoring.

In the past, the voice communication is the only way used between aircraft and ground. The defects of HF and VHF voice communication frequency congestion and effect of human factor, directly influences the efficiency and safety of flight. With the rapid development of the international aviation industry, various countries are making great efforts on implementing a variety of air-ground data link in the air traffic services (ATS) application. In 1998, the International Civil Aviation Organization (ICAO) formally proposed the establishment of new communications, navigation and surveillance (CNS) and air traffic control (ATC) system to ensure flight safety, and achieve global air traffic management [6]. Since then, the United States began to use ARINC's Aircraft Communications Addressing and Reporting System (ACARS) as air-ground data link communication mode, which is the world's most widely used air-ground data link communications system. 


\section{CURRENT STATUS OF AIR-GROUND DATA LINK}

\section{COMMUNICATIONS TECHNOLOGY RESEARCH}

Aircraft Communication Addressing and Reporting System (ACARS) was developed by ARINC company in the United States. ACARS is based on a very high frequency (VHF) airborne system and capable providing an approach for bi-directional data communication, which has the performance improvements in transmission speed, antiinterferences, low bit error rate, etc. [7]. The system consists of airborne subsystem, the ground data link service providers and ground application subsystems adopting the protocol-based on VHF digital link (VDL) characteroriented information exchange model [8], for the purpose of all kinds of information exchange. On current stage, a large number of data communications in civil aviation are implemented through the air-ground data link. Supported by addressed air-ground data communication network and satellite communications, the system achieves an integrated air-ground real-time data transmission and contributes a powerful support for the remote data exchange in flight.

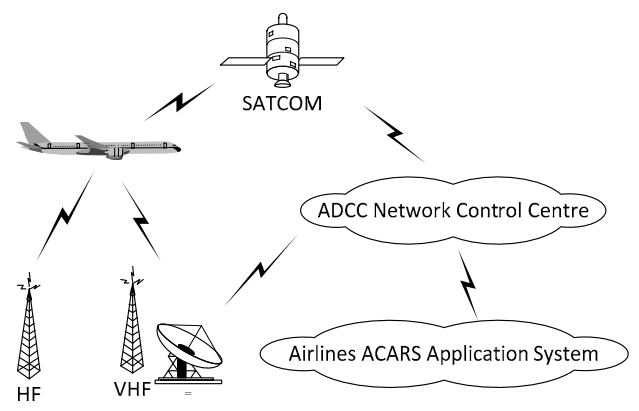

Figure 1. Schematic diagram of air-ground data link

Figure 1 shows the architecture of air-ground data link system used by China's civil aviation. In form of digital telegram, the system performs automatic transmission and exchange of information between aircraft and remote ground control station. Aircraft equipped with ACARS equipment is mainly through VHF remote ground control station (RGS) to transmit messages, and the RGS will bring the messages together to the Civil Aviation Data Center (ADCC) Network Control Center. Based on the aircraft's characteristics included in the messages, ADCC will distribute the messages to the airline that the aircraft belongs to. Ref. to Figure 2, ADCC's ground network receives ACARS signals from the aircraft in the air, and converted to the identifiable messages for airlines. Also, the network can convert the airline's messages and load them to the designated aircraft. ACARS is the core of the air-ground data communications network and the communication protocol is the key factor.

Till now, ICAO has developed four different modes of air-ground data link including VDL-A, VDL-2, VDL-3 and VDL-4 [9]. The mode A is the basis of the other three models and the fundamental communication mode, which is still used by the majority of the national and regional aviation including China civil aviation. The other three modes are the functional expansion and enhancement of the mode A, the difference mainly reflected in the transfer rate, operation frequency, encoding and communication ways [10-12]. With the increasement in the number of flights and civil aviation demand for real-time data communication, it is imperative that the modes have greater transfer rate used in the air-ground data link communication. The ICAO experts predict that the four modes will be coexist in future for a long time, basing on the fact that huge controversy still exist in the standard-setting process. The OEM has to understand the different data link modes so as to use these technologies. Although the four modes differ in some respects, but they are based on the mode A. Therefore, it is the first issue to understand the principle of VDL mode A during the process of studying air-ground data link technology.

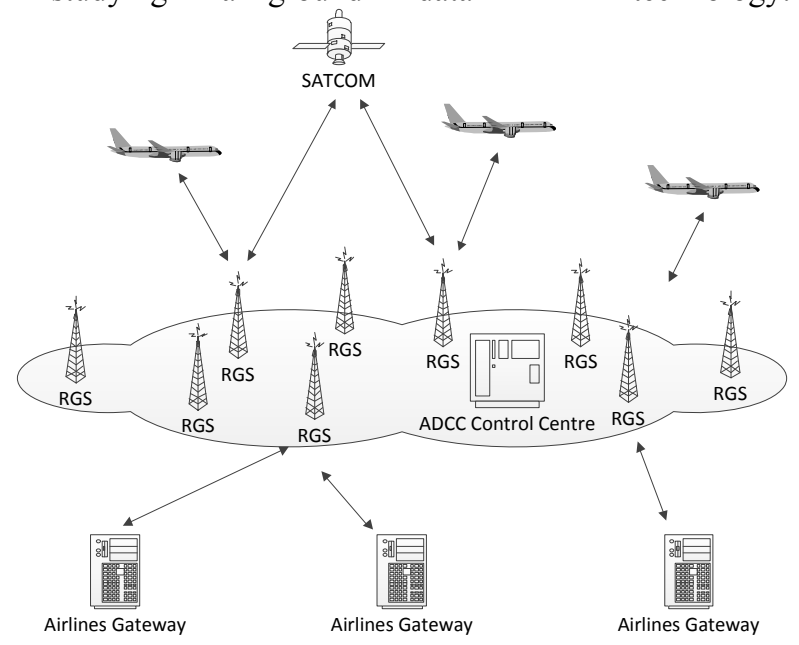

Figure 2. ACARS network diagram

In VDL-A mode, the data transmission between air and ground complies with ARINC-618 protocol strictly. Currently, only several companies can provide this kind of system. The kind of system has good decoding stability. But the data processing is in close loop and customer can only get fixed form of decoding results limiting its application in engineering, which means it cannot meet the requirement of analysis work in deeper level. However, the principal technologies in deeper level are important aspects to be grasped during the requirement allocation procedure for the systems of the large aircraft. Given the fundamental role of ACARS and current research status in this field of our country, it is undoubtedly the research work should begin from the VDL-A mode basing on ARINC-618 protocol, and the principle rules of air-ground transmission of messages should be understood comprehensively. For this purpose, the article addresses the ACARS technologies research in VDL-A mode basing on the fundamental signal analysis. 


\section{SIGNAL ACQUISITION AND ANALYSIS}

\section{A. Signal Acquisition}

Figure 3 shows the ACARS signal acquisition platform block diagram. The platform mainly includes VHF radio receiver, signal acquisition card and computer. The radio antenna receives the ACARS messages signal transmitted by the aircraft radios, then filters the signals and forwards to the 16-bit signal acquisition card, finally sends the signals to the computer and stores them for analysis. Among them, the radios are the IC-A24 receiver from ICOM company. Signal acquisition frequency was $44.1 \mathrm{KHz}$. The actual received signal unit shown in Figure 6.

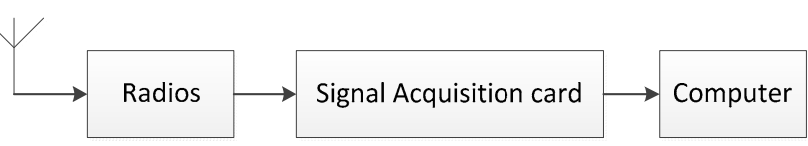

Figure 3. Signal acquisition platform block diagram

\section{B. Basic Signal Waveform Identification}

The MSK was used to modulate and demodulate the ACARS signals, which used the frequency of $2400 \mathrm{~Hz}$ and $1200 \mathrm{~Hz}$. The basic 0 and 1 signals were built through different combinations of the two frequencies. When the frequency is $2400 \mathrm{~Hz}$ waveform, one cycle of the waveform represents a binary bit. For the end of one cycle of the waveform, if the trend of the slope of the end of the waveform is positive, it is on behalf of "1"; if the trend of the slope of the end of the waveform is negative, it is on behalf of " 0 ". When the frequency is $1200 \mathrm{~Hz}$ waveform, half cycle of the waveform represents a binary bit. When the slope of end of the half-cycle waveform is positive, it is on behalf of "1"; when the slope of the half-cycle waveform is negative, it is on behalf of " 0 ". The details are shown in Figure 4.

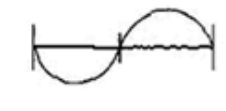

waveform indicated " 0 " in $2400 \mathrm{HZ}$

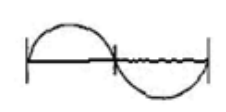

waveform indicated " 1 " in $2400 \mathrm{HZ}$

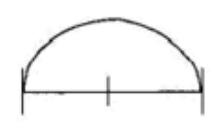

waveform indicated " 0 " in $1200 \mathrm{HZ}$
Figure 4. Binary and basic waveform in signal

According to the rules mentioned above, the decoded waveform is shown in Figure 5.

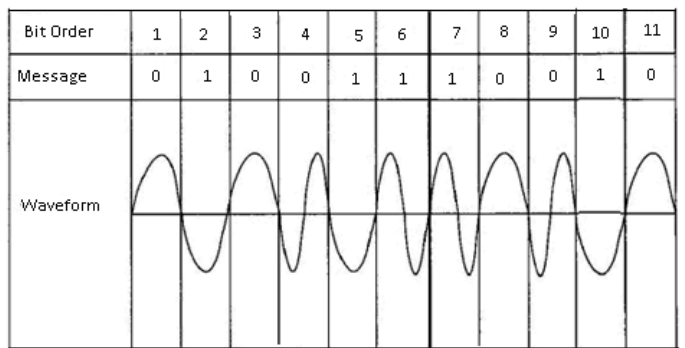

Figure 5. Waveform signal decoding

\section{Basic Signal Analysis}

Same as ordinary signals, the MSK signals were transmitted in the order of left to right. For example, a string of characters MU5131, the M was sent firstly, then U, followed by $5,1,3,1$, until the end. ACARS signals use ISO5 character set. ARINC-618 protocol provides a total of 128 characters encoding, seven bits binary number was needed to represent the encoding.

In order to improve the accuracy of the transmission, a eighth bit was added for parity check. The odd parity was used in ACARS communications, so the number of the binary code " 1 " of each character is odd in the process of signal transmission. For example, the binary encoding of the character " $\mathrm{M}$ " is " 1001101 "; In order to ensure the correctness of parity, a " 1 " is added to the binary sequence and changed to "11001101" when the message is sent.

Each character sends in sequence from low bit to high one, that is, from right to left to send. For example, the character " $M$ " is encoded to " 11001101 ", but the order of transmitting is from low bit to high one, that is " 10110011 ". The Figure 6 shows the real instance that the " $M$ " character is sending.

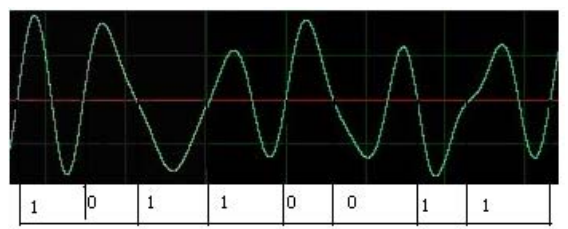

Figure 6. "M" character sending

\section{PROBLEMS AND DISCUSS}

\section{A. Instability of Signal}

In the process of the signal waveform research, the instable signal makes it difficult to analyze and process. The waveform passing through the acquisition card is not smooth, which may be caused by the weakness of the signal or interference. Its causes need a further study. For the relatively stable signals, its waveform is relatively smooth, waseshown in Figure 7.

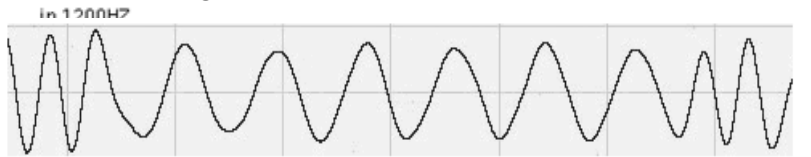

Figure 7. The desired waveform received

The waveform is not smooth enough for the relatively unstable signal, as shown in Figure 8. 


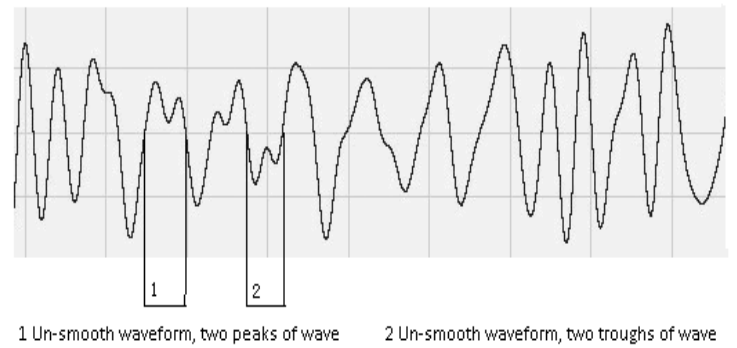

Figure 8. The un-smooth waveform received

Comparing Figure 7 and Figure 8 , it can be found that the difference is very clear between the two kind of waveform. If the signal is instable, the figure is not smooth that double peak or double bottom waveform often appears. Such differences bring difficulty to the following analysis. It is necessary to make further study in order to overcome these difficulties. In some extreme situatoions, there were some unexpected zero-crossing points, such as the bottoms in 1 and 2 of Figure 8 . This will lead to the errors in demodulation.

\section{B. Processing of Alienation Waveform}

It is found that the signals are sometimes not standard enough in the current study, which makes it difficult to analyze the signals. Between $1200 \mathrm{~Hz}$ and $2400 \mathrm{~Hz}$ frequency range, there were a large numbers of interim frequency waveform when the frequencies changed, as show in Figure 9. 1and 3were the $1200 \mathrm{~Hz}$ and $2400 \mathrm{~Hz}$ respectively. 2 was the alienation waveform which frequency was instable at $1480 \mathrm{~Hz}$ and $1820 \mathrm{~Hz}$.

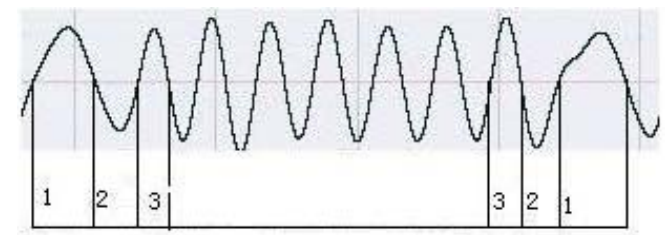

$11200 \mathrm{HZ}$ waveform, broad

2 alienation waveform, between $1200 \mathrm{HZ}$ and $2400 \mathrm{HZ}$ $32400 \mathrm{HZ}$ waveform, narrow

Figure 9. Waveform Comparison
Because a large numbers of alienation waveform exist in the process of signal waveform analysis, it makes identifying the alienation waveform as the $1200 \mathrm{~Hz}$ or $2400 \mathrm{~Hz}$ very difficult.. The statistical methods will be used to accurately classify these alienation signals; its reliability needs further verification.

\section{REFERENCES}

[1] [1] ZHANG Xue-jun; MA Yu-wen. VHF air/ground data link system and ARINC618 protocol [J]. Aviation engineering \& maintenance, 2002, (205): 41-43

[2] BI Xin-an. An analysis of the differences between the two kinds of ground-to-air data link and the system transformation [J]. Civil Aviation Economics \& Technology, 1999, 6:57-59

[3] ARINC Specification 620-3 [S]. Published: December 19, 1997.

[4] HUANG Jun-xiang. Research on ACARS Data Process [J]. Journal of Civil Aviation University of China, 2007, 2:1-3

[5] XU Chun-sheng. Application research of real-time remote monitoring technology on aircraft maintenance [J]. Journal of Civil Aviation College of China, 2003, Vol.21: 6-9

[6] GUO Xu-zhou; HUANG Sheng-guo; SUN Jian. The design and implementation of modem of VHF air-ground data link system [J]. Jiangsu Aviation, 2007,4:7-8

[7] GUO Jing. Construction, development and application of China civil aviation air/ground data chain [J]. China Civil Aviation, 2006,3, Vol.63:64-66

[8] ZHOU Jian-xing; HUANG sheng-guo; QING Li-yong. The application of VHF Portable ACARS test station [J]. Jiangsu Aviation, 2006, 2:13-14

[9] YANG Cheng-lei; HUANG Zhi-gang; Zhang-jun. ATN and VDL2 technique in CNS/ATM system [J]. Telecommunication Engineering, 2003, (3):87-91

[10] ZHANG Wei-min. VHF digital datalink communication technology analysis [J]. Navigation and Radar, 2003, (2):10-14

[11] Pereira MS, Investigation of the three competing VHF digital data link communications technology for commercial aviation [C], Digital Avionics Systems Conference, 2002. Proceedings. The 21st, 2002, $1: 3 \mathrm{C} 7 / 1-3 \mathrm{C} 7 / 8$

[12] LI Nan; CHEN Ying; XIA Yi-qiang. VDL-4: a new aviation VHF data link [J]. Telecommunication Engineering, 2003, (6):90-94

[13] ARINC Specification 618-5 [S]. Published: August 31, 2000. 